\section{Small intestinal obstruction by a Peutz-Jeghers polyp - double-balloon enteroscopic removal}

A 38-year-old woman with Peutz-Jeghers syndrome was admitted due to a 2-week history of intermittent abdominal pain and symptoms of incomplete intestinal obstruction. She had experienced symptoms of incomplete intestinal obstruction 2 years previously, and had undergone explorative laparotomy, which revealed a large polyp of the jejunum that required resection. On admission, laboratory results of the patient were unremarkable. Physical examination revealed a mild distension of the upper abdominal quadrants. Abdominal radiograph gave no hint of bowel obstruction.

Double-balloon enteroscopy via the oral route was carried out, and revealed a polyp at approximately $150 \mathrm{~cm}$ distant from the pylorus, which nearly completely occluded the intestinal lumen ( $\bullet$ Figure 1). Using guided double-balloon enteroscopy, the polyp was captured with a snare and resected by electric cautery. Resection and salvage of the polyp in one piece was achieved ( $\bullet$ Figure 2 ). No subsequent complications occurred. Histology showed a hamartoma with adenomatous components. Further examination of the remaining small bowel was refused by the patient. The patient was discharged in good health 1 day after the intervention. No intestinal symptoms reappeared in the following 12 months.

Peutz-Jeghers syndrome is characterized by multiple gastrointestinal hamartomatous polyps [1]. Peutz-Jeghers polyps have an increased risk of developing malignancy and they bear the risk of complications, such as intestinal hemorrhage or intestinal obstruction. As localization of symptomatic polyps in the small bowel prevents access by conventional gastrointestinal endoscopy, the majority of comparable cases have required surgical treatment [2].

Technical advances in endoscopy of the small bowel have been achieved by the invention of push-enteroscopy; nevertheless its range is limited [3]. Capsule endoscopy provides images from the whole small intestine [4], but lacks the ability to include therapeutic interventions, and moreover bears the potential complication of capsule impaction [5].

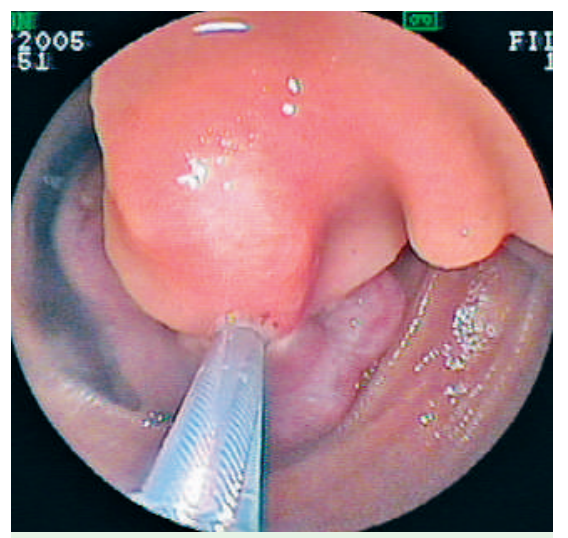

Figure 1 Double-balloon enteroscope video image of a snare-captured Peutz-Jeghers polyp in the jejunum, which nearly completely occluded the intestinal lumen.

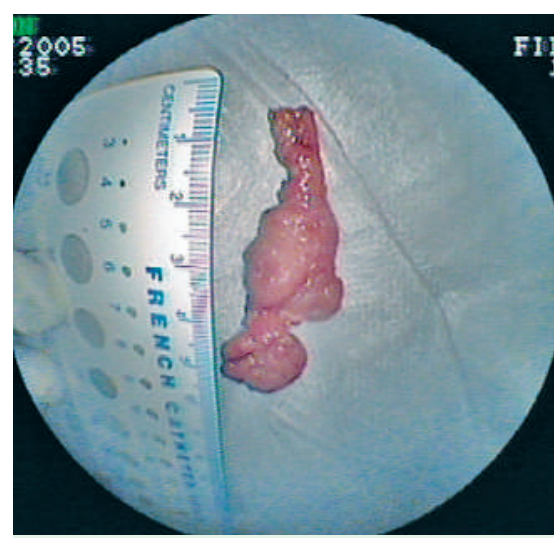

Figure 2 Resected Peutz-Jeghers polyp of the jejunum, measuring $25 \mathrm{~mm}$ (width) $\times$ $50 \mathrm{~mm}$ (length). Histopathology showed a hamartoma with adenomatous components.

The recent invention of double-balloon enteroscopy [6] has improved greatly the ability to image the small intestine; moreover it enables endoscopically directed therapy to be carried out.

In the present clinically subacute setting, recurrence of a symptomatic Peutz-Jeghers polyp was likely. In order to avoid surgical re-intervention, double-balloon enteroscopy was carried out as a firstline diagnostic procedure. This proved to be a successful minimally invasive diagnostic and therapeutic method, accurately visualizing the polyp and allowing effective, definitive, and safe therapy by polypectomy. This report points out the beneficial role of double-balloon enteroscopy in Peutz-Jeghers syndrome with symptomatic polyps of the small intestine, potentially obviating the need for surgical intervention.

\section{Endoscopy_UCTN_Code_CCL_1AC_2AC}

\section{H. Ullerich, C. Maaser, W. Domschke, T. Kucharzik}

Department of Medicine B, University of Muenster, Muenster, Germany

\section{References}

1 McGarrity TJ, Kulin HE, Zaino RJ. Peutz-Jeghers syndrome. Clinical reviews. Am J Gastroenterol 2000; 95: 596-604

2 Spigelman AD, Arese P, Phillips RKS. Polyposis: the Peutz-Jeghers syndrome. Br J Surg 1995; 82: 1311 - 1314

3 Davies GR, Gertner DJ, Benson MJ et al. Diagnostic, therapeutic and practical advantages of small bowel examination with a push type enteroscope. Gut 1995; 37: $346-$ 352

4 Caspary $R$, von Falkenhausen M, Krautmacher $C$ et al. Comparison of capsule endoscopy and magnetic resonance imaging of polyps of the small intestine in patients with familial adenomatous polyposis or with Peutz-Jeghers syndrome. Endoscopy 2004; 36: 1054-1059

5 Jonnalagadda S, Prakesh C. Intestinal strictures can impede wireless capsule enteroscopy. Gastrointest Endosc 2003; 57: $418-$ 420

6 Yamamoto H, Sekine Y, Sato Y et al. Total enteroscopy with a nonsurgical steerable double-balloon method. Gastrointest Endosc 2001; 53: 216-220

\section{Bibliography}

DOI 10.1055/s-2007-966417

Endoscopy 2007; 39: E193

(c) Georg Thieme Verlag KG Stuttgart · New York . ISSN 0013-726X

\section{Corresponding author}

\section{H. Ullerich, MD}

Department of Medicine B

Westfaelische Wilhelms-Universitaet Muenster Albert-Schweitzer-Str. 33

D-48129 Muenster

Germany

Fax: +49-251-8347576

ullerih@mednet.uni-muenster.de 